\title{
Photoionization of highly charged carbonlike ions
}

\author{
Sultana N. Nahar and Anil K. Pradhan \\ Department of Astronomy, Ohio State University, Columbus, Ohio 43210 \\ (Received 1 November 1991)
}

\begin{abstract}
Close-coupling calculations using the $\boldsymbol{R}$-matrix method have been carried out for the photoionization cross sections of carbonlike ions: $\mathrm{F}^{3+}, \mathrm{Ne}^{4+}, \mathrm{Na}^{3+}, \mathrm{Mg}^{6+}, \mathrm{Al}^{7+}, \mathrm{Si}^{8+}, \mathrm{S}^{10+}, \mathrm{Ar}^{12+}, \mathrm{Ca}^{14+}$, and $\mathrm{Fe}^{20+}$, which are of interest in the calculation of plasma opacities. This is an extension of previous work for radiative data for the $\mathbf{C}$ sequence. Detailed results including extensive structures due to autoionizing resonances are presented for the ground states; however, the calculations have also been carried out for a large number of excited bound states of each ion and their features are discussed. The partial photoionization cross sections of the ground state into excited states of the residual ion are obtained. The effect of resonances on the branching ratios of partial cross sections at excited thresholds is discussed. Interesting features related to the $Z$ dependence of the ground-state photoionization cross sections of all ions in the $C$ sequence are discussed.
\end{abstract}

PACS number(s): $32.80 . \mathrm{Fb}$

\section{INTRODUCTION}

The calculation of plasma opacities requires a huge amount of radiative data for the bound-bound and bound-free transitions for a large number of bound states in all constituent atoms and ions. In order to determine accurate astrophysical opacities, under the auspices of the Opacity Project $[1,2]$, we have carried out extensive computations for both bound-bound and bound-free transitions along isoelectronic sequences for $Z=1-14,16,18$, 20 , and 26 in the close-coupling $(\mathrm{CC})$ approximation using the $R$-matrix method. The results for the photoionization cross sections and oscillator strengths are also useful for many other plasma applications. As most of the computed data are of much higher accuracy than achieved heretofore (i.e., prior to the Opacity Project), the results may serve as benchmark values for comparison with experiment or other theoretical calculations. However, because of the extent of the data computed, only a very small fraction of it can be presented in publications, and attempts are under way to make it available in archival form. With particular reference to photoionization cross sections, although these cross sections have been computed for several hundred bound states of each atom and ion, at typically 1000 or 2000 energies for each bound-state cross section, we present only the photoionization cross sections for the ground state and a few selected excited states that are expected to be of primary interest in applications, as well as for comparison with other studies of ions along isoelectronic sequences.

In our previous works [3-5], the photoionization cross sections and oscillator strengths for $\mathrm{C}^{0}, \mathrm{~N}^{+}$, and $\mathrm{O}^{2+}$ and a few other ions have been reported. The present work completes the $\mathrm{C}$ sequence, from $\mathrm{F}^{3+}$ up to $\mathrm{Fe}^{20+}$, of ions that are astrophysically important. In addition, the present report is a study of the photoionization cross sections along an isoelectronic sequence. To our knowledge no previous works have reported such an analysis, in the $\mathrm{CC}$ approximation, for more than two or three ions [6].
Results are given for the ground-state photoionization cross sections with emphasis on the following four points: (i) autoionization structures due to resonances, (ii) partial cross sections for photoionization into the ground and various excited states of the residual ion, (iii) effect of resonances on the branching ratios at excited threshold energies of the residual ion, and (iv) the $Z$ scaling of the cross sections. Particular attention is directed toward partial cross sections; these are important in the analysis of emission spectra from plasmas, with a photoionizing radiation source, as they determine the level populations under conditions that are not local thermodynamic equilibrium (non-LTE). The photoionization cross section of an excited state is given as an example of some of the features observed for excited states.

\section{COMPUTATIONS}

The $\mathrm{CC}$ approximation involves the wave-function expansion of the $(e+i o n)$ system in terms of the eigenfunctions of the target or the residual "core" ion. With the exception of $\mathrm{C}^{0}$, where a larger configuration-interaction (CI) basis is needed to represent the $\mathrm{C}^{+}$target states, the eigenfunction expansion for the remainder of the $\mathrm{C}$ sequence consists of the eight states $2 s^{2} 2 p\left({ }^{2} P^{\circ}\right)$, $2 s 2 p^{2}\left({ }^{4} P,{ }^{2} D,{ }^{2} S,{ }^{2} P\right)$, and $2 p^{3}\left({ }^{4} S^{\circ},{ }^{2} D^{\circ},{ }^{2} P^{\circ}\right)$ of the $n=2$ complex. The spectroscopic or principal configurations that dominate the target states are $2 s^{2} 2 p, 2 s 2 p^{2}$, and $2 s 2 p^{3}$, and the correlation configurations in the CI expansion for the target states are $2 s^{2} 3 s, 2 s^{2} 3 p, 2 s^{2} 3 d, 2 s 2 p 3 s$, $2 s 2 p 3 p, 2 s 2 p 3 d, 2 p^{2} 3 s, 2 p^{2} 3 p, 2 p^{2} 3 d$, and $2 s 3 d^{2}$. For the $(e+i o n)$ system, the calculated energies of the target states are replaced with the observed values for ions from $\mathrm{F}^{4+}$ to $\mathrm{Si}^{9+}$, while for ions from $\mathrm{S}^{10+}$ to $\mathrm{Fe}^{20+}$ the energies are the calculated ones [4], since many of these states are not observed. In all cases the difference between the calculated and experimental energies of the target states does not exceed a few percent [4].

The autoionization resonances belonging to the thresh- 
olds of excited states of the target ion are resolved in the same manner as described in detail in Ref. [5], with two kinds of energy mesh for the tabulation of the photoionization cross sections. For the calculation of accurate recombination-rate coefficients, the resonances need to be resolved fully. The low-lying, relatively broad resonances are resolved with a very fine, constant energy interval and the higher ones with a constant interval of effective quantum number so as to delineate the narrower resonances corresponding to the higher members of the Rydberg series. However, for the partial cross sections, a constant mesh in effective quantum number is used for the entire energy range. Photoionization of all the bound states of C-like ion configurations $2 s^{2} 2 p^{2}, 2 s 2 p^{3}, 2 s^{2} 2 p n l, 2 s 2 p^{2} n l$, $2 s^{2} 3 s n l, 2 s^{2} 3 p n l$, and $2 p^{3} n l$ with $n=n_{\max } \leq 10$ and $l=l_{\text {max }} \leq 3$, are considered.

\section{RESULTS}

Total photoionization cross sections of the ground and a large number of excited bound states for the ions of the $C$ sequence have been calculated. Analogously to experiment, the present method is enable to determine the total number of bound states of an atom or ion. The energies of the bound states are the eigenvalues of the Hamiltonian of the $(N+1)$ electron system, where $N$ is the number of electrons in the residual ion. Table $I$ lists the total number of bound states considered for each ion. As the ionic charge increases along the sequence, more of the autoionization states become bound because of stronger nuclear attraction that moves the resonances down in energy as $Z^{2}$. Thus one needs to take into account the photoionization of an increasing number of bound states, along the isoelectronic sequence, for a given set of $n_{\max }$ and $l_{\max }$ (as defined above).

\section{A. Ground-state photoionization cross sections}

The ground-state photoionization cross sections of the ions are presented in the lowest panels of Figs. I (a) $-1(j)$, and values of the threshold cross sections $\sigma_{0}$ of the ground state are presented in Table $I$. The results of $\mathbf{C}^{0}$, $\mathrm{N}^{+}$, and $\mathrm{O}^{2+}$ are also presented in the table for completeness. These results are taken from Ref. [5]. In Figs. $1(a)-1(j)$, the presence of a large number of autoionizing resonances can be observed. The near-threshold autoionization resonances can influence the threshold cross sections $\sigma_{0}$ significantly, as can be seen for the cases of $\mathrm{Ne}^{4+}, \mathrm{Na}^{5+}, \mathrm{Si}^{8+}$, and $\mathrm{Fe}^{20+}$. Comparison with the other calculations (e.g., Ref. [7]) shows significant differences in the threshold cross sections. For example, the values of $\sigma_{0}$ obtained by Henry [7] are 12.19, 6.65, 3.65, and $1.40 \mathrm{Mb}$ for $\mathrm{C}^{0}, \mathrm{~N}^{+}, \mathrm{O}^{2+}$, and $\mathrm{Ne}^{4+}$, respectively, whereas the present values are $18.10,8.22,2.59$ (taken from Ref. [5]), and $1.57 \mathrm{Mb}$ for the same ions, respectively. The calculations by Henry were carried out in the close-coupling approximation; a limited amount of coupling was considered, and no autoionization was taken into account. The presence of resonances can influence the background cross sections as well. Although the background gets smoother with higher energies, the near-threshold region may show variations as in the cases for $\mathrm{Ne}^{4+}, \mathrm{Na}^{5+}, \mathrm{Al}^{7+}, \mathrm{Si}^{8+}, \mathrm{S}^{10+}, \mathrm{A}^{12+}, \mathrm{Ca}^{14+}$, and $\mathrm{Fe}^{20+}$

Another earlier work on the carbon isoelectronic sequence was carried out in the high-energy range by Chen [8] for the ground configuration $1 s^{2} 2 s^{2} 2 p^{2}$. Chen employed a relativistic multiconfiguration Dirac-Fock approximation considering only the configuration interaction within the $n=2$ complex. The exchange interaction between the continuum and bound states was excluded, and no autoionization was considered in that work. We cannot compare directly with Chen's results since the present $\mathrm{CC}$ calculations are in the relatively low-energy range, whereas the calculation of Chen's cross sections starts at energies higher than in this work. However, an interesting finding of Chen's results is that the relativistic effects do not play a significant role in the photoionization process, even for very highly charged ions (e.g., less than $14 \%$ for $\mathrm{Xe}^{48+}$ ). Therefore the present results for ions up to $\mathrm{Fe}^{20+}$ should not be affected by the neglect of relativistic effects, and intermediate coupling is not necessary.

\section{B. Excited-state photoionization cross sections}

As mentioned earlier, because of the large volume of data, photoionization cross sections for the excited states

TABLE I. Ionization energy $E_{\mathrm{tP}}(\mathrm{Ry})$, threshold cross section $\sigma_{0}(\mathrm{Mb})$ into the ground state ${ }^{2} P^{\circ}$, and total number $M$, the theoretically determined number of bound states of C-like ions corresponding to configurations $2 s^{2} 2 p^{2}, 2 s 2 p^{3}, 2 p^{4}, 2 s^{2} 2 p n l, 2 s 2 p^{2} n l$, and $2 p^{3} n l$, with $n \leq 10$ and $l \leq 3$. Photoionization cross sections are obtained for all these individual states. "Calc." represents the calculated value of $E_{\mathrm{IP}}$ and "Expt." represents the observed value from Ref. [9]. An asterisk next to $\sigma_{0}$ indicates presence of a resonance at the threshold. The notation $a[b]$ for a value means $a \times 10^{6}$.

\begin{tabular}{|c|c|c|c|c|c|c|c|c|c|}
\hline \multirow[b]{2}{*}{ Ion } & \multicolumn{2}{|c|}{$E_{\mathrm{IP}}$} & \multirow[b]{2}{*}{$\sigma_{0}$} & \multirow[b]{2}{*}{$M$} & \multirow[b]{2}{*}{ Ion } & \multicolumn{2}{|c|}{$E_{\text {IP }}$} & \multirow[b]{2}{*}{$\sigma_{0}$} & \multirow[b]{2}{*}{$M$} \\
\hline & Calc. & Expt. & & & & Calc. & Expt. & & \\
\hline$C^{0}$ & 0.845 & 0.828 & $1.81[1]$ & 159 & $\mathrm{Al}^{7+}$ & 20.91 & 20.96 & $6.28[-1]$ & 369 \\
\hline $\mathrm{N}^{+}$ & 2.18 & 2.18 & 8.22 & 171 & $\mathrm{Si}^{8+}$ & 25.79 & 25.87 & $1.14^{*}$ & 389 \\
\hline $\mathrm{O}^{2+}$ & 4.04 & 4.04 & $2.59^{*}$ & 194 & $\mathbf{s}^{10+}$ & 37.05 & & $2.67[-1]$ & 469 \\
\hline$F^{3+}$ & 6.40 & 6.41 & 2.56 & 231 & $A^{12+}$ & 50.32 & & $4.10[-1]$ & 524 \\
\hline $\mathrm{Ne}^{4+}$ & 9.27 & 9.29 & $1.57^{*}$ & 256 & $\mathrm{Ca}^{14+}$ & 65.59 & & $2.06[-1]$ & 574 \\
\hline $\mathrm{Na}^{9+}$ & 12.65 & 12.67 & $4.05^{*}$ & 311 & $\mathrm{Fe}^{20+}$ & 123.40 & & $1.07[-1]^{*}$ & 656 \\
\hline $\mathbf{M g}^{6+}$ & 16.53 & 16.57 & $7.51[-1]$ & 343 & & & & & \\
\hline
\end{tabular}



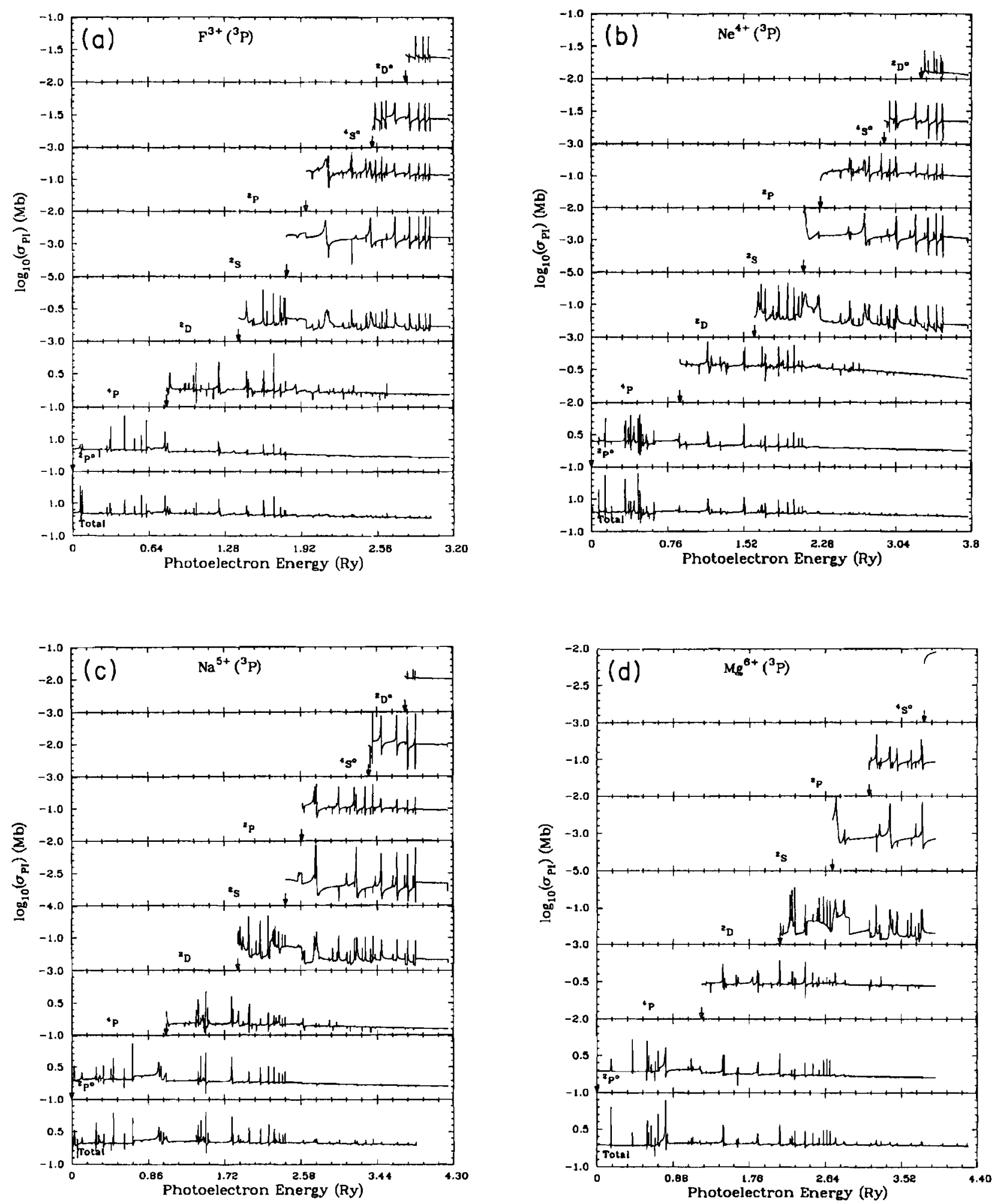

FIG. 1. (a) Total (bottom panel) and partial (panels above) photoionization cross section of ground state $2 s^{2} 2 p^{23} P$ of $F^{3+}$. The partial cross sections correspond to leaving the residual ion in states $2 s^{2} 2 p\left({ }^{2} P o\right), 2 s 2 p^{2}\left({ }^{4} P,{ }^{2} D,{ }^{2} S,{ }^{2} P\right)$, and $2 p^{3}\left({ }^{4} S^{o_{1}}{ }^{2} D^{o}\right)$. (b) Same as (a) except for ion $\mathrm{Ne}^{4+}$. (c) same as (a) except for ion $\mathrm{Na}^{3+}$. (d) Same as (a) except for ion $\mathrm{Mg}^{6+}$. (e) Same as (a) except for ion $\mathrm{Al}^{7+}$. (f) Same as (a) except for ion $\mathrm{Si}^{8+}$. (g) Same as (a) except for ion $\mathrm{S}^{10+}$. (h) Same as (a) except for ion $\mathrm{Ar}^{12+}$. (i) Same as (a) except for ion $\mathrm{Ca}^{14+}$. (j) Same as (a) except for ion $\mathrm{Fe}^{20+}$. 

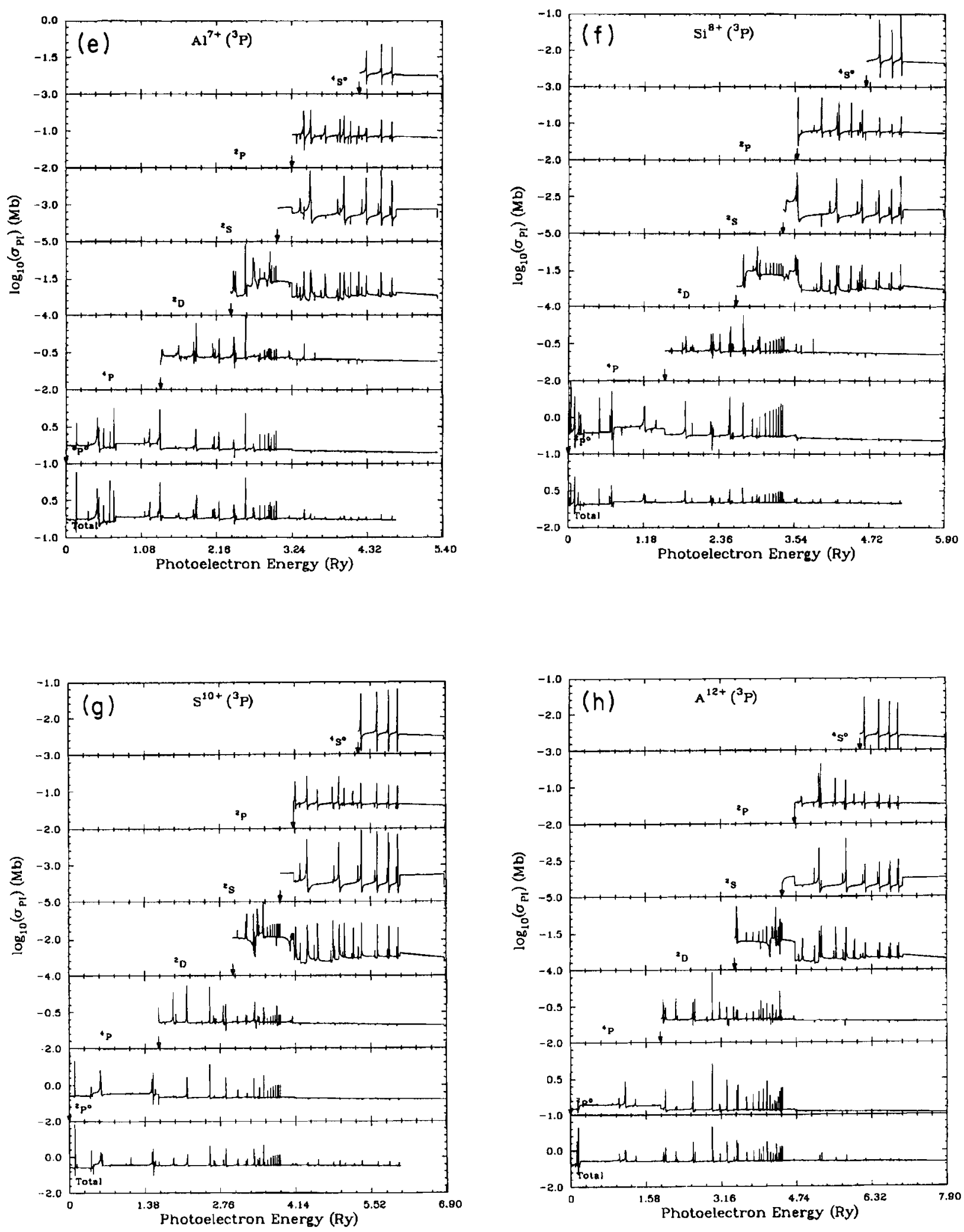

FIG. 1. (Continued). 

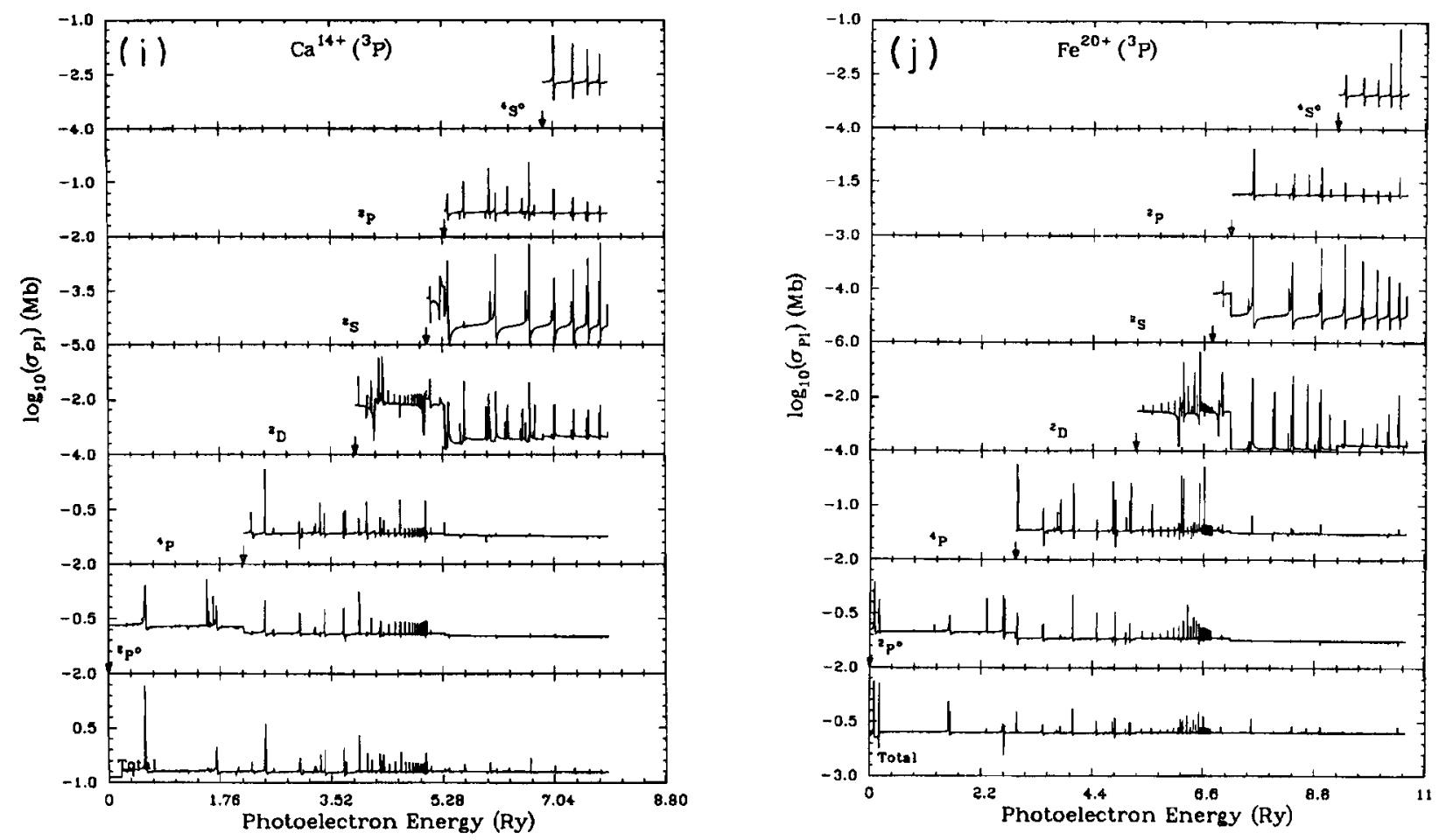

FIG. 1. (Continued).

(except one example) are not presented here. However, all of the results will be used to obtain recombination-rate coefficients for excited states and for total (e +ion) recombination in subsequent work [5]. There are three important features of excited-state cross sections that we should like to mention: (1) indirect photoionization through autoionizing states alone, (2) strong resonances due to dipole transitons between core states of the residual ion or photoexcitation of core (PEC), and (3) nonhydrogenic behavior.

By "indirect photoionization" we mean photoionization of equivalent electron excited states, e.g., photoionization of $1 s^{2} 2 p^{4}\left({ }^{3} P\right)$, in the energy range where direct photoionization of the optical $2 p$ electron is not accessible by the incident photon, i.e., below the $1 s^{2} 2 p^{3}\left({ }^{4} S^{\circ},{ }^{2} D^{\circ},{ }^{2} P^{\circ}\right)$ states of the residual ion and above the ionization threshold $2 s^{2} 2 p\left({ }^{2} P^{\circ}\right)$. In this region the photoionization proceeds only through autoionizing states converging onto the $1 s^{2} 2 p^{3}$ states and there is no "background" cross section as usual. The photoionization is thus the result of coupling among residual ion states, but it is forbidden in approximations that neglect such coupling. However, the effective cross sections may be very large as a result of the autoionizing resonances. Examples are given in Refs. [3] ad [5].

Although we do not give the results here, we might note that, as in the case of the first three elements of the sequence [5], the photoionization cross sections of each C-like ion in the present work show PEC resonances for the photoionization of excited bound states of a Rydberg series. The PEC resonances occur at incident photon en- ergies that correspond to the energy differences between states of the target ion related by a dipole transition, e.g., at energies corresponding to transitions $2 s^{2} 2 p\left({ }^{2} P^{\circ}\right) \rightarrow 2 s 2 p^{2}\left({ }^{2} D,{ }^{2} S,{ }^{2} P\right)$ in the boronlike residual ions.

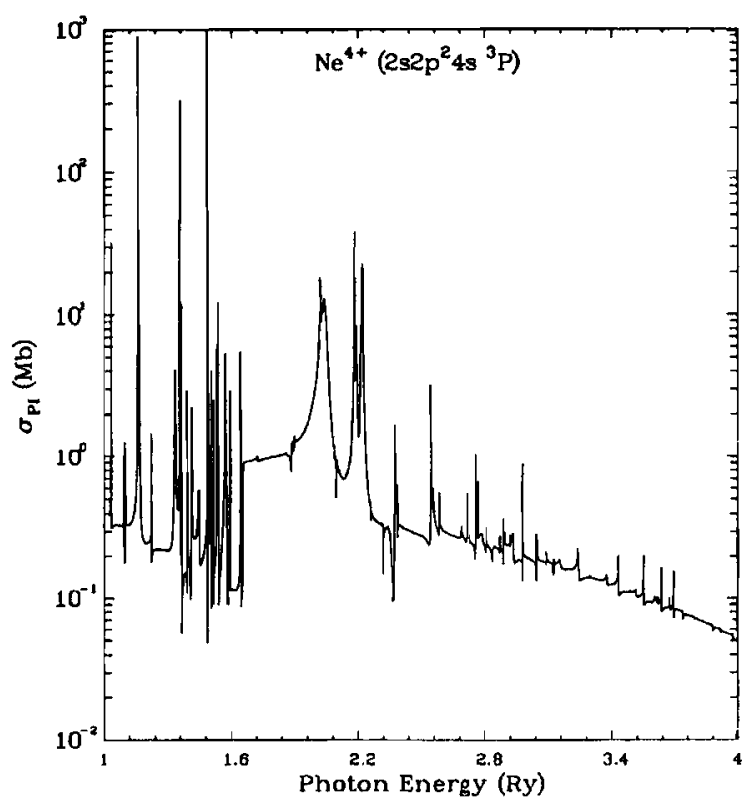

FIG. 2. Photoionization cross section of excited $2 s 2 p^{2} 4 s^{3} P$ state of $\mathrm{Ne}^{4+}$. 
Excited states that are apparently hydrogenic may be affected by resonances and show large variations in the background cross sections. Figure 2 shows such an example for the excited state $2 s 2 p^{2} 4 s^{3} \mathrm{P}$ of $\mathrm{Ne}^{4+}$. Excitedstate cross sections may also be very much larger than the ground-state cross sections and in general are found to have considerably enhanced cross sections compared with the hydrogenic or other simpler approximations such as the central-field model.

\section{Partial cross sections}

The $\mathrm{CC}$ approximation readily enables one to calculate the partial cross sections for photoionization into various states of the residual ion. The partial photoionization cross sections of the ${ }^{3} \boldsymbol{P}$ ground state of $\mathrm{C}$-like ions into various states of the residual B-like ions are obtained and presented in Figs. 1(a)-1(j). The states of the residual ion (i.e., the eigenstates in the $\mathrm{CC}$ expansion) are $2 s^{2} 2 p\left({ }^{2} P^{\circ}\right), 2 s 2 p^{2}\left({ }^{4} P,{ }^{2} D,{ }^{2} S,{ }^{2} P\right)$, and $2 p^{3}\left({ }^{4} S^{\circ},{ }^{2} D^{\circ},{ }^{2} P^{\circ}\right)$. In Figs. 1(a) $-1(j)$, each panel, except the lowest one, shows partial cross section corresponding to a particular target state as specified, and the arrows point to the threshold energies of the target states with respect to the ground state. In these figures the small differences in resonance profiles near threshold between the total groundstate cross section (lowest panel) and the partial cross section into the ${ }^{2} P^{\circ}$ state (the second lowest panel) region of the ions arise because of the difference in mesh sizes used to resolve the resonances for the total and partial cross sections.

Figures $1(a)-1(j)$ show that the background cross section for the photoionization into an excited state of the target is in general much smaller compared with that into the target ground state, yet it may show the presence of large number of resonances. Resonances can enhance the background significantly for photoionization into certain excited states of the residual ion. This can be observed particularly for photoionization into the ${ }^{2} D$ state of the target for all the ions from $\mathrm{F}^{3+}$ to $\mathrm{Fe}^{20+}$. As the ${ }^{2} D$ state also corresponds to the resonance transition in boronlike systems, we expect that the intensity of the uv transition ${ }^{2} D-{ }^{2} P^{\circ}$ may be enhanced. Resonance structures in the partial cross sections are also of special interest since they might affect the branching ratios considerably (discussed in Sec. III D). In general, they are of crucial importance in the precise determination of level populations under conditions of non-LTE in laboratory and astrophysical plasmas; the latter includes the modeling of astronomical sources such as planetary nabulae, active galactic nuclei, and stellar atmospheres. As we have considered a number of excited states of the photoionized B-like ions, it should help construct fairly complete and accurate nonLTE models of the emergent spectra, including some cascading effects.

The partial photoionization into the ${ }^{4} P$ and ${ }^{2} D$ states is of particular interest since lines from these levels are prominently observed in the ultraviolet spectra of plasma sources $[10,11]$. The intensity ratio of the resonance to the intercombination lines, $R=I\left({ }^{2} D-{ }^{2} P^{\circ}\right) / I\left({ }^{4} P-{ }^{2} P^{\circ}\right)$, is valuable in the temperature and density diagnostics since the ${ }^{2} D$ level corresponds to the resonance transition and the ${ }^{4} P$ is the metastable state. In optically thin plasmas, these lines are excited by a collision due to electron impact; however, in the presence of a radiation source, the photoionization process is a prime contributor. The range of wavelengths in B-like ions for the dipole-allowed transition ${ }^{2} D \rightarrow{ }^{2} P^{0}$, is from $654.6 \AA$ for $F^{4+}$ to $173.5 \AA$ for $\mathrm{Fe}^{20+}$ and, for the intercombination transition ${ }^{4} P \rightarrow{ }^{2} P^{\circ}$, is from $1158 \AA$ for $\mathrm{F}^{3+}$ to $316.7 \AA$ for $\mathrm{Fe}^{20+}$. Thus, together with the related work on electron-impact excitation of B-like ions [11], the results reported here should be helpful in the analysis of observations in the extreme uv, in the range specified, according to the ionization stage under consideration.

\section{Branching among excited thresholds}

As the partial photoionization cross sections have been obtained for a number of excited thresholds of the residual ion, the branching ratios for photoionization into these states, $\sigma_{0 i} / \sigma_{0}$, may be calculated in detail. These are often of interest in the analysis of experiments or observations where the rates of populating excited states, relative

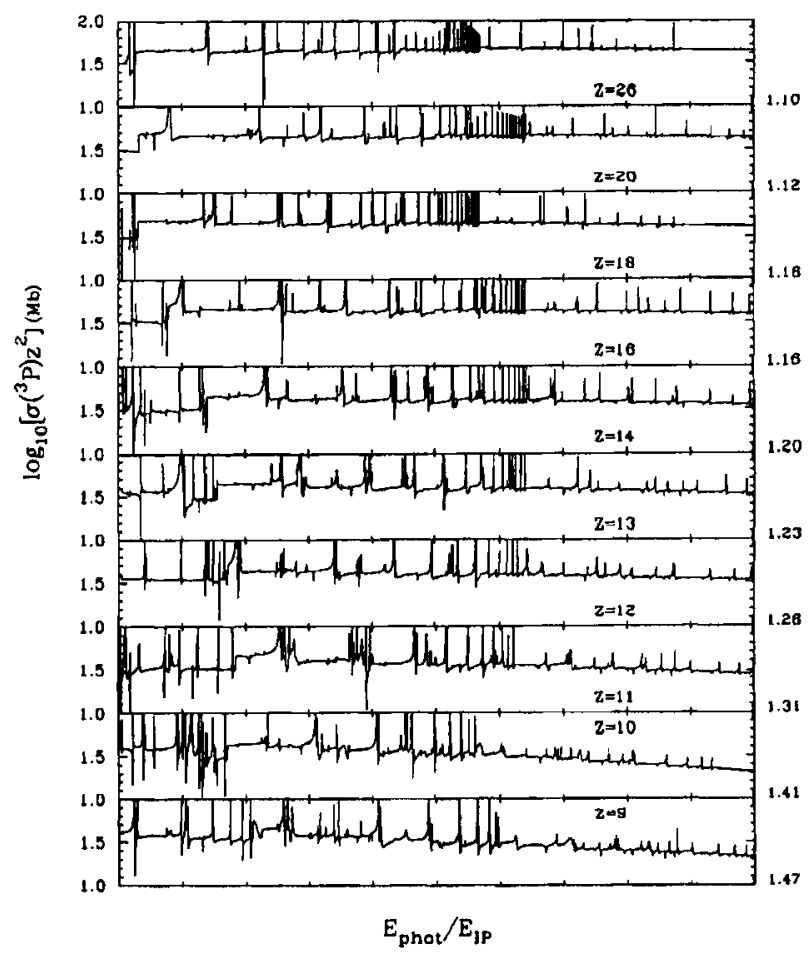

FIG. 3. Photoionization cross sections (in Mb), scaled with the ionic charge $z(=Z-6)$, of the ${ }^{3} P$ ground state of the $C$ isoelectronic sequence from $Z=9$ to 26 . Except for the topmost panel, upper limits of the panels are not given. The photon energy (in $R y$ ) is scaled by the ionization potential. For each panel the energy range goes from 1.0, that is, from the binding energy of the ion, to a little over the photon energy corresponding to the highest target threshold. The highest energy is printed on the right side of each panel. 
to the ground state, are needed. Of particular importance are the metastable states, which might contain a significantly large population following photoionization at photon frequencies where the metastable state is accessible. However, the partial cross sections, as given in Fig. 1, show extensive resonance structures, which may introduce considerable variations in the branching ratios. With reference to photoionization into the ground state ${ }^{2} P^{\circ}$ versus the metastable state ${ }^{4} P$, while one may readily discern the general trend that the ${ }^{2} P^{\circ}$ cross sections decreases more rapidly for all ions in the sequence, a precise calculation of branching ratios at a given energy needs great care. In fact, since the resonances dominate most of the near-threshold energy ranges, it is advisable to specify the energy range where the branching ratio might be needed in a particular case, e.g., an experimental beamwidth or the ionizing radiation field with plasma linewidth, such that an energy average may be carried out. Otherwise, in the vicinity of a resonance, the ratio may be highly uncertain.

\section{E. $\mathbf{Z}$ dependence of the cross sections}

While simpler methods such as the hydrogenic or central-field model employ a straightforward $Z$ dependence of the photoionization cross sections at the threshold, the more detailed cross sections in the $C C$ approximation, which contain extensive autoionization structures, are expected to show a more complicated behavior along an isoelectronic sequence. It can be noted that the threshold cross sections $\sigma_{0}$ from $C^{0}$ to $\mathrm{Fe}^{20+}$ in Table I cannot be formulated to fit a simple $Z$-scaled form because of variations introduced by the coupling of states and autoionizing resonances. In the table an asterisk next to a $\sigma_{0}$ value indicates the presence of a resonance at the threshold.

However, the average background cross section may show more consistent behavior. In Fig. 3 we study the $Z$-scaled pattern of the ground-state photoionization cross sections, along the sequence, for $Z=9-26$. We plot $\log _{10}\left[z^{2} \sigma\left({ }^{3} P ; E\right)\right]$ versus $E / E_{I P}$, where $z$ is the ionic charge, $E$ is the photon energy, and $E_{I P}$ is the ionization potential (presented in Table 1). The energy range for each panel goes from $E / E_{I P}=1.0$ to $E_{t \max } / E_{I P}$, is the photon energy corresponding to energy of the highest threshold of the residual ion. We find that, although the cross sections are rich in resonance structures (as we have seen earlier), the $Z$-scaled values of the cross section and photon energy are relatively smoothly varying across the resonances; (i.e., the background photoionization cross sections show remarkably small variations.

The following numerical values are obtained for the $Z$ scaled background cross sections for the photoionization of the ground state of the C-sequence ions: $\log _{10}\left(z^{2} \sigma\right) \simeq 1.6$ for $Z=7-18, \simeq 1.3$ for $Z=6$, and 1.8 for $Z=20,26$. Furthermore, for the entire range of photon energies considered, the values of $\log _{10}\left[z^{2} \sigma(E)\right]$ lie between 1.3-1.8 for all ions. The apparently small variations in the $\boldsymbol{Z}$-scaled cross sections are nonetheless significant and may be attributed to coupling effects that manifest themselves differently for each ion.

\section{CONCLUSION}

Photoionization cross sections of highly charged ions of the carbon isoelectronic sequence have been calculated in the close-coupling approximation. Detailed cross sections for a large number of bound states are obtained for each ion; results are given for the photoionization of the ground state, except for one excited state as an example. Owing to the presence of extensive autoionizing resonances in the cross sections, it is not possible to obtain a simple $\mathbf{Z}$-scaling or extrapolation formula for the threshold cross sections, although one may discern a relatively slow variation in the background cross sections. For the same reason, the branching ratios of partial cross sections are state dependent and do not follow any given pattern. The present results should be useful in the diagnostics and modeling of far-uv plasma sources under non-LTE conditions.

\section{ACKNOWLEDGMENTS}

This work was supported by the National Science Foundation (AST-8996215). S.N.N. is also the recipient of support from the College of Mathematical and Physical Sciences at the Ohio State University. The computational work was carried out at the Ohio Supercomputer Center in Columbus, $\mathbf{O H}$.
[1] M. J. Seaton, J. Phys. B 20, 6363 (1987); A. K. Pradhan, Phys. Scr. 35, 840 (1987).

[2] K. A. Berrington, P. G. Burke, K. Butler, M. J. Seaton, P. J. Storey, K. T. Taylor, and Yu Yan, J. Phys. B 20, 6379 (1987).

[3] D. Luo, A. K. Pradhan, H. E. Saraph, P. J. Storey, and Y. Yu, J. Phys. B 22389 (1989).

[4] D. Luo and A. K. Pradhan, J. Phys. B. 22, 3377 (1989).

[5] Sultana N. Nahar and Anil K. Pradhan, Phys. Rev. A 44, 2935 (1991).

[6] A. K. Pradhan, Phys. Rev. A 25, 592 (1982). This is a study of the ground-state photoionization of three ions in the $\mathrm{Li}$ sequence $\mathrm{C}^{3+}, \mathrm{N}^{4+}$, and $\mathrm{O}^{5+}$.

[7] R. J. W. Henry, Astrophys. J. 161, 1153 (1970).

[8] M. H. Chen, Phys. Rev. A 35, 4586 (1987).

[9] C. E. Moore, Atomic Energy Levels, Natl. Bur. Stand. (U.S.) Circ. No. 467 (U.S. GPO, Washington, DC, 1949).

[10] M. Hayes and H. Nussbaumer, Astron. Astrophys. 134, 193 (1984).

[11] R. D. Blum and A. K. Pradhan, Astrophys. J. Suppl. (to be published). 